\title{
Una metanalisi che non risolve i nostri dubbi
}

\author{
Commento all'articolo: "Medical management to prevent recurrent nephrolithiasis in adults: \\ a systematic review for an American College of Physicians Clinical Guideline”
}

\section{Piergiorgio Messa}

Direttore U.O. di Nefrologia, Dialisi e Trapianto Renale, Fondazione IRCCS Ca' Granda, Ospedale Maggiore Policlinico, Milano

È usuale per i lettori della bibliografia medica imbattersi ciclicamente in articoli che rivisitino argomenti rimasti critici quanto indefiniti nel tempo.

La revisione sistematica, pubblicata recentemente da Fink et al (1), appartiene a questa tipologia di articoli, affrontando gran parte dei quesiti irrisolti riguardo agli interventi terapeutici indirizzati alla prevenzione della nefrolitiasi (NL) ricorrente.

In particolare, gli Autori, sulla base dei lavori di maggior livello di evidenza disponibili, affrontano tre tra i principali quesiti che rimangono irrisolti:

- Esistono interventi preventivi e, se sì, quali tra essi sono efficaci nella prevenzione della nefrolitiasi ricorrente?

- È utile effettuare lo studio metabolico di base e/o l'analisi della natura del calcolo per indirizzare l'intervento profilattico?

- Vi sono dati che giustifichino la valutazione delle modificazioni metaboliche indotte dagli interventi per avere una predizione dell'efficacia preventiva?

Nei prossimi paragrafi, dopo una breve sintesi di quanto riportato dagli Autori, mi limiterò ad alcune considerazioni personali su ogni singolo punto.

\section{Efficacia degli interventi preventivi}

Gli Autori hanno analizzato separatamente l'efficacia degli interventi dietetici e di quelli farmacologici. Tra i primi, le uniche evidenze riportate come moderatamente solide riguarderebbero l'efficacia dell'aumento dell'introduzione idrica, mentre le evidenze su altri tipi di intervento (riduzione delle proteine animali, riduzione dell'introito salino, modificazioni dell'apporto calcico, riduzione dell'eccesso di ossalato) risulterebbero contraddittorie. D'altra parte, per quanto riguarda l'efficacia degli interventi farmacologici, la maggior parte di questi (tiazidico, citrato, allopurinolo), sia quando utilizzati da soli che in combinazione tra di loro, sembrerebbero aggiungere un vantaggio sulla prevenzione della NL al solo intervento dietetico.

Nella valutazione di questi, come di tutti gli altri risultati, è da precisare che il livello della qualità degli studi disponibili è globalmente modesto. Inoltre la durata di questi studi è quasi sempre relativamente breve. Quest'ultimo aspetto introduce numerose problematicità. La prima è relativa alla scarsità degli eventi registrabili in un follow-up breve, il che potrebbe dare ragione della mancata significatività statistica nel confronto di efficacia tra il braccio di intervento e quello di controllo. Inoltre, visto che tutti gli interventi farmacologici erano sovrapposti ad indicazioni dietetiche, non è facile scorporare l'entità dell'efficacia nei due bracci in un periodo di osservazione breve. Infatti mentre nelle fasi iniziali degli interventi consigliati l'adesione ai consigli dietetici è usualmente abbastanza elevata, con il prolungamento dell'osservazione, la compliance alla dieta è spesso destinata a ridursi, a fronte del mantenimento di una più prolungata adesione alla terapia farmacologica. Quindi un'analisi degli eventi effettuata su periodi più lunghi potrebbe permettere di cogliere differenze non evidenziabili in studi relativamente brevi.

D'altro canto, è anche da osservare che la brevità degli studi non permette neppure di valutare in modo esaustivo la potenziale tossicità nel lungo termine di tutti gli interventi terapeutici in particolare farmacologici.

Quindi la non conclusività di molti di questi studi, oltre che ad una modesta qualità del disegno, è da ascriversi alla ridotta durata dell'osservazione.

\section{Utilità dello studio metabolico prima degli esami}

Dagli studi riportati dagli Autori risulterebbe di scarsa o nulla utilità sia lo studio della composizione chimica del calcolo che lo studio metabolico dei fattori di rischio litogeno. Il messaggio che indirettamente potrebbe derivare da questo articolo è che non vi è alcuna utilità ad eseguire queste indagini nel paziente con NL.

Questa conclusione va però riconsiderata alla luce di alcune considerazioni.

In primo luogo, è utile sottolineare che la diagnosi di alcuni tipi di NL (cistina, acido urico), non prese in considerazione da questa revisione, si giova certamente dello studio della composizione chimica del calcolo. 
Inoltre, lo studio metabolico e l'analisi chimica del calcolo non sono finalizzati solo a definire il tipo di intervento preventivo, ma anche ad escludere tutte le possibili cause di NL, tra cui quelle endocrine (iperparatiroidismo innanzi tutto), oltre che ad aiutare a porre diagnosi di alcune forme rare di calcolosi.

Infine, e lo dico per un'affezione personale ad una nostra antica linea di ricerca nella quale si dava particolare risalto ad alcuni aspetti di connessione tra NL, interventi dietetici e metabolismo osseo (2-4), lo studio metabolico della NL è utile anche per dare uno sguardo ad alcuni aspetti metabolici che coinvolgono l'apparato scheletrico, la cui conoscenza è utile nella gestione della terapia dietetica e farmacologica, in particolare nelle pazienti con NL di sesso femminile, maggiormente esposte al rischio di osteoporosi.

\section{Valutazione delle variazioni biochimiche dopo trattamento e relazione con gli outcome}

Ancor minore sembrerebbe essere il peso informativo delle variazioni biochimiche ottenute in conseguenza dei differenti trattamenti riguardo il verificarsi degli eventi litiasici.

Sulla relativa affidabilità di questa affermazione pesa ancora di più lo scarso livello degli studi disponibili.

Certamente ritengo che la necessità di un certo livello di monitoraggio nel tempo dei dati biochimici in pazienti in trattamento serva non solo a verificare se il trattamento sta agendo in modo efficace nel controllo quantomeno dei dati biochimici che si avrebbe intenzione di correggere, ma anche a monitorare i potenziali effetti iatrogeni di tali interventi.

In conclusione, questa revisione serve a dare conferma di alcune (poche) certezze che già avevamo, lasciando purtroppo aperti la maggior parte dei dubbi che continueremo ad avere.

Spesso il risultato di queste rivisitazioni periodiche di argomenti non frequentati da tutti ha un doppio effetto: rassicurante, in quanto conferma che quanto si fa nella pratica quotidiana è ancora valido, ma sconfortante perché sottolinea come non vi siano di fatto nuove acquisizioni che permettano interventi di maggior successo rispetto a quelli che da anni continuiamo a mettere in atto. In genere queste considerazioni si concludono con la solita frase: c'è bisogno di nuovi studi, disegnati con maggiore appropriatezza statistica, eseguiti su un numero più elevato di pazienti, con tempi più prolungati ecc... ma crediamo veramente che questo possa avvenire?... qui ci vorrebbe più tempo e un altro lungo dibattito

Dichiarazione di conflitto di interesse: L'Autore dichiara di non avere conflitto di interessi.

Contributi economici agli Autori: L'Autore dichiara di non avere ricevuto sponsorizzazioni economiche per la preparazione dell'articolo.

Indirizzo dell'Autore:

Prof. Piergiorgio Messa

Direttore U.O. di Nefrologia, Dialisi e Trapianto Renale

Fondazione IRCCS Ca' Granda

Ospedale Maggiore Policlinico

20122 Milano

pmessa@policlinico.mi.it

\section{Bibliografia}

1. Fink HA, Wilt TJ, Eidman KE, et a. Medical management to prevent recurrent nephrolithiasis in adults: a systematic review for an American College of Physicians Clinical Guideline. Ann Intern Med 2013; 158(7): 535-43.

2. Messa P, Mioni G, Montanaro D, et al. About a primitive osseous origin of the so-called 'renal hypercalciuria'. Contrib Nephrol 1987; 58: 106-10.

3. Messa P, Mioni G, Paganin L, Cruciatti A, Greco PL, Turrin D. Urinary citrate, bone resorption and intestinal alkali absorption in stone formers with fasting hypercalciuria. Scanning Microsc 1994; 8(3): 531-8; discussion 538-9.

4. Messa P, Marangella M, Paganin L, et al. Different dietary calcium intake and relativesupersaturation of calcium oxalate in the urine of patients forming renal stones. Clin Sci (Lond) 1997; 93(3): 257-63. 